\title{
BOWEN-RUELLE MEASURES FOR CERTAIN PIECEWISE HYPERBOLIC MAPS
}

$\mathrm{BY}$

LAI-SANG YOUNG ${ }^{1}$

\begin{abstract}
We consider a class of piecewise $C^{2}$ Lozi-like maps and prove the existence of invariant measures with absolutely continuous conditional measures on unstable manifolds
\end{abstract}

This work is a small step forward in the following program: Suppose a compact neighborhood is mapped into itself and the map displays some chantic behavior. Is there a strange attractor, or more specifically, is there a Bowen-Ruelle measure? Axiom A systems aside, current techniques have hardly begun to provide answers to these questions. One of the purposes of this note is to demonstate, in a very limited way, how certain 1-dimensional results can sometimes be useful in handling dissipative systems in 2-dimension.

The 1-dimensional result we alluded to says that piecewise expanding endomorphisms of the unit interval have smooth invariant measures [LY]. What we prove here is an analogous statement for certain piecewise hyperbolic attractors in 2-dimension. The prime examples that motivate this study are the Lozi maps, though our analysis has little to do with the precise nature of the equations studied by Lozi or Misiurewicz $[\mathbf{M}]$. Our main result is that these "generalized Lozi maps" have invariant measures with absolutely continuous conditional measures on unstable manifolds. As a consequence they have Bowen-Ruelle measures.

We begin by isolating several properties of the Lozi maps. They are essentially the properties upon which our proof depends. Maps satisfying these hypotheses will henceforth be called "generalized Lozi maps". (See Figure 1.)

Let $R=[0,1] \times[0,1]$ and let $f: R \rightarrow R$ be a continuous injective map. We assume that $f$ or some iterate of $f$ takes $R$ into its interior. Let $0<a_{1}<\cdots<a_{q}<1$ and let $S=\left\{a_{1}, \ldots, a_{q}\right\} \times[0,1]$. We assume that $f \mid(R-S)$ is a $C^{2}$ diffeomorphism onto its image with $|\operatorname{Jac}(f)|<1$ and that both $f \mid(R-S)$ and $f^{-1} \mid f(R-S)$ have bounded second derivative. We further impose the following conditions on $f \mid(R-S)$ (geometric interpretations are given in parentheses).

$$
\operatorname{Inf}\left\{\left(\left|\frac{\partial f_{1}}{\partial x}\right|-\left|\frac{\partial f_{1}}{\partial y}\right|\right)-\left(\left|\frac{\partial f_{2}}{\partial x}\right|+\left|\frac{\partial f_{2}}{\partial y}\right|\right)\right\} \geq 0
$$

( $D f$ preserves cones making $<45^{\circ}$ with the $x$-axis),

$$
\operatorname{Inf}\left\{\left|\frac{\partial f_{1}}{\partial x}\right|-\left|\frac{\partial f_{1}}{\partial y}\right|\right\}=u>1
$$

Received by the editors November 15, 1982.

1980 Mathematics Subject Classification. Primary 58F15.

Key words and phrases. Hyperbolicity, unstable manifolds, invariant measures.

${ }^{1}$ Research partially supported by NSF. 


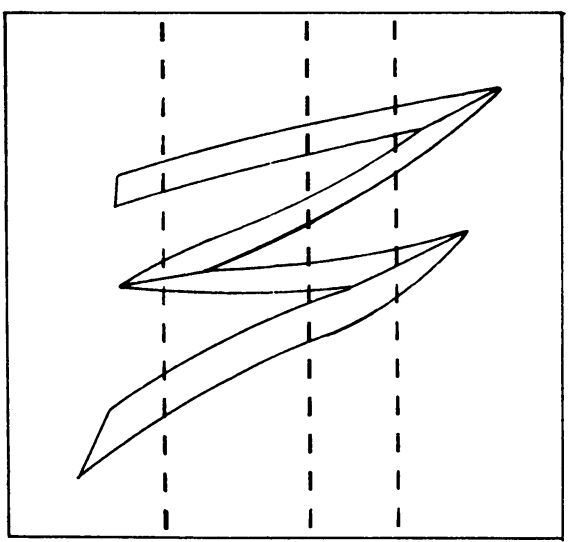

FIGURE 1. Example of a "generalized Lozi map"

(restricted to these cones the action of $D f$ when projected onto the $x$-axis is uniformly expanding),

$$
\operatorname{Sup}\left\{\frac{\left|\partial f_{1} / \partial y\right|+\left|\partial f_{2} / \partial y\right|}{\left(\left|\partial f_{1} / \partial x\right|-\left|\partial f_{1} / \partial y\right|\right)^{2}}\right\}<1
$$

(horizontal expansion dominates the action of $D f$ on vertical vectors), and

$$
\exists N \in \mathbf{Z}^{+} \text {s.t. } u^{N}>2 \text { and } f^{k} S \cap S=\emptyset \text { for } 1 \leq k \leq N
$$

( $f$ expands horizontally more than it folds).

Note that (H4) is vacuous when $u>2$. (Hence Figure 1 is legitimate.) Note also that these hypotheses are indeed satisfied by many Lozi maps. Lozi maps are usually given by

$$
L(x, y)=(1+y-a|x|, b x) .
$$

Changing coordinates this can be written as

$$
f(x, y)=(1+b y-a|x|, x) .
$$

It is easy to verify that for open intervals of $a$ and $b, f$ takes some square $[c, c] \times[c, c]$ into itself and satisfies (H1)-(H4). ${ }^{2}$

DEFINITION 1. A Borel probability measure $\mu$ on $R$ is said to have absolutely continuous conditional measures on unstable manifolds if there exist measurable partitions $P_{1} \subset P_{2} \subset \cdots$ of $R$ and measurable sets $V_{1} \subset V_{2} \subset \cdots$ s.t.

1. $\mu V_{n} \uparrow 1$ as $n \rightarrow \infty$,

2. each element of $P_{n} \mid V_{n}$ is an open subset of some unstable manifold and

3. if $\left\{\mu_{c}: c \in P_{n} \mid V_{n}\right\}$ denotes the system of conditional measures on elements of $P_{n} \mid V_{n}$, and $m_{c}$ denotes Riemannian measure on $c$, then for almost every $c \in \mathcal{P}_{n} \mid V_{n}$, we have $\mu_{c} \ll m_{c}$.

We now state our main result.

\footnotetext{
${ }^{2}$ As this manuscript was being written I learned that P. Collet and Y. Levy had jointly obtained a result similar to ours for certain parameter values of the Lozi map [CL].
} 
THEOREM. If $f: R \rightarrow R$ is a generalized Lozi map, then $f$ has an invariant Borel probability measure $\mu$ s.t.

1. Local unstable manifolds exist at $\mu$-a.e. point and

2. $\mu$ has absolutely continuous conditional measures on unstable manifolds.

The idea of our proof is as follows: Observe that unstable manifolds (when they exist) are piecewise smooth curves zigzagging across $R$ (turning around at random places. Our strategy is first to construct an invariant measure $\mu$ that behaves nicely on neighborhoods of the singularity set $S$. This is done following a combination of the methods used by Sinai $[\mathbf{S}]$ and Lasota and Yorke [LY]. Since all turns are created by passing through $S$, we now have control over their impact as well. In particular, this allows us to construct a noninvariant measure $\tilde{\mu}$ equivalent to $\mu$ on an arbitrarily large set and having the property that its conditional measures on unstable manifolds are (obviously) absolutely continuous. This finishes the proof.

The following notations will be used: The map $p: R \rightarrow[0,1]$ denotes projection onto the first factor. Lebesgue measure on $[0,1]$ is denoted by $m$. For $g:[a, b] \rightarrow \mathbf{R}$, $\bigvee_{a}^{b} g$ denotes the total variation of $g$ on $[a, b]$. If $\mu$ is a measure on $R$, then $f_{*} \mu$ is given by $f_{*} \mu(E)=\mu\left(f^{-1} E\right)$. If $J \subset[0,1]$ is a closed interval and $\alpha: J \rightarrow[0,1]$ is a $C^{2}$ function with $\left|\alpha^{\prime}\right| \leq 1$, then $f(\operatorname{graph}(\alpha))$ is a union of finitely many smooth curves (H1). We denote them by $\left\{L_{i}(\alpha)\right\}$, dropping the $\alpha$ whenever no ambiguity arises. For $k>1$, denote the smooth segments of $f^{k} \operatorname{graph}(\alpha)$ by $\left\{L_{i_{1} \cdots i_{k}}\right\}$, where the indices are chosen so that $f L_{i_{1} \cdots i_{k}}=\bigcup_{j} L_{i_{1} \cdots i_{k} j}$. Let $\mu_{0}$ be the measure on $\operatorname{graph}(\alpha)$ s.t. $p_{*} \mu_{0}=$ normalized Lebesgue measure on $J$. For $k=1,2, \ldots$, define $\mu_{k}=\left(f^{k}\right)_{*} \mu_{0}$. From (H1) and (H2) we know that for each $i_{1} \cdots i_{k}, p_{*} \mu_{k} \mid L_{i_{1} \cdots i_{k}}$ is absolutely continuous to $m$. We denote its density by $g_{i_{1} \cdots i_{k}}$ and the density of $p_{*} \mu_{k}$ by $\hat{g}_{k}$. That is, we have $\sum_{i_{1} \cdots i_{k}} g_{i_{1} \cdots i_{k}}=\hat{g}_{k}$. When it is convenient to consider $f^{N}$ instead of $f$, we will write

$$
f^{N k} \operatorname{graph}(\alpha)=\bigcup_{i_{1} \cdots i_{k}} L_{i_{1} \cdots i_{k}}^{(N)}
$$

and

etc.

$$
d\left(p_{*} \mu_{N k} \mid L_{i_{1} \cdots i_{k}}^{(N)}\right)=g_{i_{1} \cdots i_{k}}^{(N)} d m
$$

LEMMA. Under the hypotheses of the theorem, there exists an invariant Borel probability measure $\mu$ and a function $g:[0,1] \rightarrow[0, \infty)$ of bounded variation s.t. $d\left(p_{*} \mu\right)=g d m$.

ProOF. Fix $J \subset[0,1]$ and a $C^{2}$ function $\alpha: J \rightarrow[0,1]$ with $\left|\alpha^{\prime}\right| \leq 1$. We will show that $\exists M$ s.t. $\bigvee_{0}^{1} \hat{g}_{k} \leq M$ for all $k$. This will imply that $\bigvee_{0}^{1}\left(n^{-1} \sum_{k=1}^{n} \hat{g}_{k}\right) \leq M$, and since $\int_{0}^{1}\left(n^{-1} \sum_{k=1}^{n} \hat{g}_{k}\right) d m=1$ the sequence $\left\{n^{-1} \sum_{k=1}^{n} \hat{g}_{k}\right\}_{n=1,2, \cdots}$ is precompact in $L^{1}([0,1], m)$. Choose a subsequence $\left\{n_{i}\right\}$ s.t. as $i \rightarrow \infty,\left(n_{i}\right)^{-1} \sum_{k=1}^{n_{i}} \mu_{k}$ converges in the weak star topology to a Borel probability measure $\mu$ and

$$
\frac{1}{n_{i}} \sum_{k=1}^{n_{i}} \hat{g}_{k} \stackrel{L^{1}}{\rightarrow} \text { some function } g .
$$

It follows immediately that $\mu$ is invariant, $d\left(p_{*} \mu\right)=g d m$ and that $\bigvee_{0}^{1} g \leq M$. 

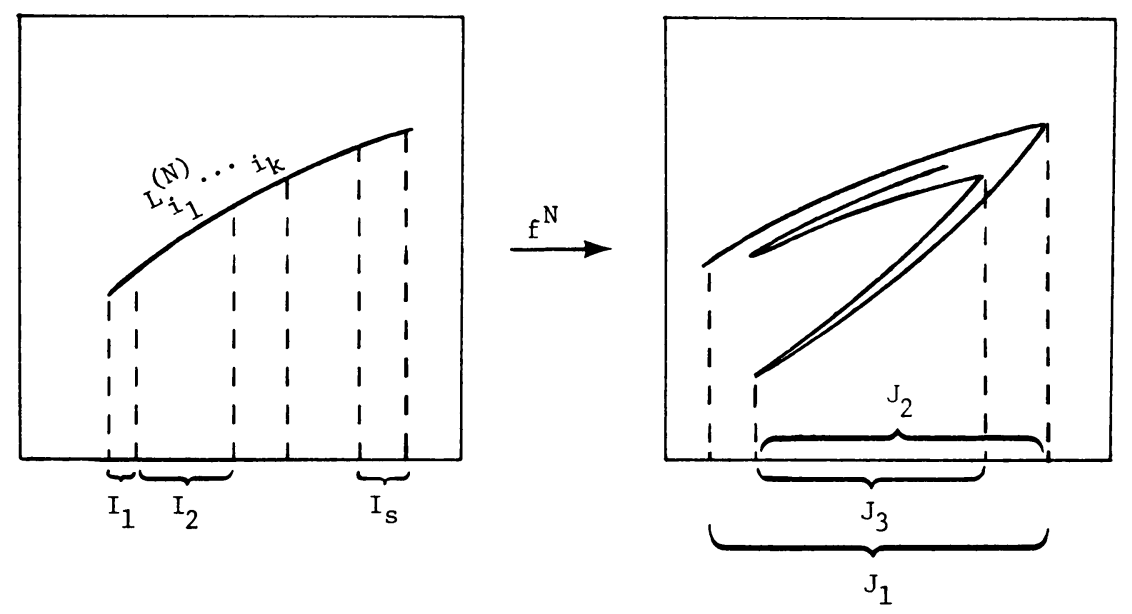

FIGURE 2

Let $N \in \mathbf{Z}^{+}$satisfy (H4). It suffices to show the uniform boundedness of $\bigvee_{0}^{1} \hat{g}_{N k}$ for $k=1,2, \ldots$ We will prove that $\exists A$ s.t. for every $i_{1} \cdots i_{k}$,

$$
\sum_{j} \bigvee_{0}^{1} g_{i_{1} \cdots i_{k} j}^{(N)} \leq A \int_{0}^{1} g_{i_{1} \cdots i_{k}}^{(N)} d m+\frac{2}{u^{N}} \bigvee_{0}^{1} g_{i_{1} \cdots i_{k}}^{(N)}
$$

Then if $\beta_{k}=\sum_{i_{1} \cdots i_{k}} \bigvee_{0}^{1} g_{i_{1} \cdots i_{k}}^{(N)}$, we would have

$$
\beta_{k+1}=\sum_{i_{1} \cdots i_{k}}\left(\sum_{j} \bigvee_{0}^{1} g_{i_{1} \cdots i_{k} j}^{(N)}\right) \leq A+\frac{2}{u^{N}} \beta_{k}
$$

so that for all $k$,

$$
\bigvee_{0}^{1} g_{N k} \leq \beta_{k} \leq A \sum_{i=0}^{\infty}\left(\frac{2}{u^{N}}\right)^{i}<\infty
$$

We now fix $i_{1} \cdots i_{k}$ and prove $(*)$. Let $p L_{i_{1} \cdots i_{k}}^{(N)}=\bigcup_{j=1}^{s} I_{j}$, where $\left\{f^{N}(x, y): x \in\right.$ $\left.I_{j}\right\}=L_{i_{1} \cdots i_{k} j}^{(N)}$ and $J_{j}=p L_{i_{1} \cdots i_{k} j}^{(N)}$. Define $\tilde{f}_{j}: I_{j} \rightarrow J_{j}$ by $\tilde{f}_{j}(x)=p \circ f^{N}(x, y)$ for $(x, y) \in L_{i_{1} \cdots i_{k}}^{(N)}$. Then each $\tilde{f}_{j}$ is a $C^{2}$ diffeomorphism between $I_{j}$ and $J_{j}$ with $\left|\tilde{f}_{j}^{\prime}\right| \geq u^{N}((\mathrm{H} 1),(\mathrm{H} 2))$ and $\left|\left(\tilde{f}_{j}^{-1}\right)^{\prime \prime}\right| \leq Q$ for some universal $Q$. (This follows from (H3) by a direct computation.) Now

$$
\begin{aligned}
\sum_{j} \bigvee_{0}^{1} g_{i_{1} \cdots i_{k} j}^{(N)}= & \sum_{j} \underbrace{\underbrace{}_{J_{j}}\left[\left(g_{i_{1} \cdots i_{k}}^{(N)} \circ \tilde{f}_{j}^{-1}\right)\left|\left(\tilde{f}_{j}^{-1}\right)^{\prime}\right|\right]}_{\text {(1) }} \\
& +\sum_{j} \underbrace{\left[\left|\tilde{f}_{j}^{\prime}\left(l_{j}\right)\right|^{-1} g_{i_{1} \cdots i_{k}}^{(N)}\left(l_{j}\right)+\left|\tilde{f}_{j}^{\prime}\left(r_{j}\right)\right|^{-1} g_{i_{1} \cdots i_{k}}^{(N)}\left(r_{j}\right)\right]}_{\text {(2) }},
\end{aligned}
$$


where $l_{j}$ and $r_{j}$ denote the left and right end points of $I_{j}$ respectively. Consider one $j$ at a time.

$$
\begin{aligned}
& \bigvee_{J_{j}}\left(g_{i_{1} \cdots i_{k}}^{(N)} \circ \tilde{f}_{j}^{-1}\right)\left|\left(\tilde{f}_{j}^{-1}\right)^{\prime}\right|=\int_{J_{j}}\left|\left(\left(g_{i_{1} \cdots i_{k}}^{(N)} \circ \tilde{f}_{j}^{-1}\right)\left(\tilde{f}_{j}^{-1}\right)^{\prime}\right)^{\prime}\right| d m \\
& \quad=\int_{J_{j}}\left|\left(g_{i_{1} \cdots i_{k}}^{(N)} \circ \tilde{f}_{j}^{-1}\right)^{\prime}\right|\left|\left(\tilde{f}_{j}^{-1}\right)^{\prime}\right| d m+\int_{J_{j}}\left(g_{i_{1} \cdots i_{k}}^{(N)} \circ \tilde{f}_{j}^{-1}\right)\left|\left(\tilde{f}_{j}^{-1}\right)^{\prime \prime}\right| d m \\
& \quad \leq \frac{1}{u^{N}} \bigvee_{I_{j}} g_{i_{1} \cdots i_{k}}^{(N)}+\frac{Q}{u^{N}} \int_{I_{j}} g_{i_{1} \cdots i_{k}}^{(N)} d m
\end{aligned}
$$

so that

$$
\begin{gathered}
\text { (1) }+\left|\tilde{f}_{1}^{\prime}\left(l_{1}\right)\right|^{-1} g_{i_{1} \cdots i_{k}\left(l_{1}\right)}^{(N)}+\left|\tilde{f}_{s}^{\prime}\left(r_{s}\right)\right|^{-1} g_{i_{1} \cdots i_{k}}^{(N)}\left(r_{s}\right) \\
\leq \frac{1}{u^{N}} \bigvee_{0}^{1} g_{i_{1} \cdots i_{k}}^{(N)}+\frac{Q}{u^{N}} \int_{0}^{1} g_{i_{1} \cdots i_{k}}^{(N)} d m .
\end{gathered}
$$

Now we claim that there is a universal $d>0$ s.t. the points $0, l_{2}, \ldots, l_{s}, 1$ are pairwise at least $d$ apart. We have $\left|0-l_{2}\right|,\left|l_{s}-1\right| \geq d$ because we may assume that $f^{N k} R \subset$ int $R$. That $\left|r_{j}-l_{j}\right| \geq$ some $d$ for $j=2, \ldots, s-1$ follows from (H4). Thus if $I=[l, r]$ is either $\left[0, l_{1}\right]$ or $\left[l_{s}, 1\right]$ or $\left[l_{j}, r_{j}\right], j=2, \ldots, s-1$, then

$$
\begin{aligned}
g_{i_{1} \cdots i_{k}}^{(N)}(l)+g_{i_{1} \cdots i_{k}}^{(N)}(r) & \leq 2\left(\min _{I} g_{i_{1} \cdots i_{k}}^{(N)}\right)+\bigvee_{I} g_{i_{1} \cdots i_{k}}^{(N)} \\
& \leq \frac{2}{d} \int_{I} g_{i_{1} \cdots i_{k}}^{(N)} d m+\bigvee_{I} g_{i_{1} \cdots i_{k}}^{(N)}
\end{aligned}
$$

Note that $g_{i_{1} \cdots i_{k}}^{(N)}(0)=g_{i_{1} \cdots i_{k}}^{(N)}(1)=0$, so that the terms in (2) not accounted for in $(* *)$

$$
\leq \frac{1}{u^{N}} \frac{2}{d} \int_{0}^{1} g(N)_{i_{1} \cdots i_{k}} d m+\frac{1}{u^{N}} \bigvee_{0}^{1} g_{i_{1} \cdots i_{k}}^{(N)}
$$

Thus

$$
\text { (1) }+(2) \leq\left(Q+\frac{2}{d}\right) \frac{1}{u^{N}} \int_{0}^{1} g_{i_{1} \cdots i_{k}}^{(N)} d m+\frac{2}{u^{N}} \bigvee_{0}^{1} g_{i_{1} \cdots i_{k}}^{(N)}
$$

which completes the proof of $(*)$ and the lemma.

Let $W_{\delta}^{u}(x)$ denote the local unstable manifold at $x$ (assuming it exists) s.t. $p W_{\delta}^{u}(x)=[p(x)-\delta, p(x)+\delta]$. Implicit in this notation is the assertion that $W_{\delta}^{u}(x)$ contains no cusps. Let $D(S, \delta)$ denote the $\delta$-neighborhood of $S$.

PROOF OF THEOREM. If $\mu$ is any invariant probability measure with $d\left(p_{*} \mu\right)=$ $g d m$ for some bounded $g$, say $g \leq M_{0}$, then for $\delta>0$,

$$
\sum_{k=0}^{\infty} \mu\left(f^{k} D\left(S, \delta u^{-k}\right)\right)=\sum_{k=0}^{\infty} \mu D\left(S, \delta u^{-k}\right) \leq 2 \delta M_{0} \sum_{k=0}^{\infty} u^{-k}<\infty
$$

By the Borel-Cantelli lemma, $\mu$-a.e. $x$ is in $f^{k} D\left(S, \delta u^{-k}\right)$ for at most finitely many $k$. That is, for $\mu$-a.e. $x, \exists \delta(x)>0$ s.t. $f^{-k} x \notin D\left(S, \delta(x) u^{-k}\right)$ for all $k>0$. This implies the existence of $W_{\delta(x)}^{u}(x)$. (For more details see [KS].) Let us fix 
one measure $\mu$ constructed as in the lemma with $\operatorname{graph}(\alpha)=W_{\delta(x)}^{u}$ for some $x$. This guarantees that the $L_{i_{1} \cdots i_{k}}(\alpha)$ will not cross other unstable manifolds. For $\delta>0$, let $\Lambda_{\delta}=\left\{x \in R: d\left(f^{-k} x, S\right) \geq \delta u^{-k} \forall k \geq 0\right\}$. Then each $\Lambda_{0}$ is closed and $\lim _{\delta \rightarrow 0} \mu \Lambda_{\delta}=1$.

We now define a sequence of measurable partitions $P_{1} \subset P_{2} \subset \cdots$. All notations will be as in Definition 1. For $n \in \mathbf{Z}^{+}$, let $\left\{U_{1}, \ldots, U_{2^{n}}\right\}$ be the partition of $R$ into $2^{n}$ vertical columns of width $2^{-n}$. For $x \in U_{i} \cap \Lambda_{2^{-n}}$, let $c(x)=W_{2^{-n}}^{u}(x) \cap U_{i}$. Let $V_{n}=\bigcup_{x \in \Lambda_{2-n}} c(x)$ and $P_{n}=\left\{c(x): x \in V_{n}\right\} \cup\left\{R-V_{n}\right\}$. It suffices to show that for every $\varepsilon>0$ and every $n$, there is a set $\tilde{V}_{n} \subset V_{n}$ s.t. $\mu \tilde{V}_{n}>\mu V_{n}-\varepsilon$ and condition 3 in Definition 1 is satisfied when $\mu$ is replaced by $\chi_{\tilde{V}_{n}} \mu$. In fact, for given $\varepsilon$ and $n$, we will construct a noninvariant measure $\tilde{\mu}$ and a set $\tilde{V}$ with $\mu \tilde{V}>1-\varepsilon$ s.t. $\tilde{\mu} \ll \mu$, is equivalent to $\mu$ on $\tilde{V}$, and satisfies condition 3 with respect to $P_{n^{\prime}}$ for some $n^{\prime} \geq n$. It is straightforward to verify that this implies the desired result.

Now let $n \in \mathbf{Z}^{+}$and $\varepsilon>0$ be given. Let $n^{\prime}$ be a large number to be determined later and let $\tilde{U}_{1}, \ldots, \tilde{U}_{2^{n^{\prime}}}$ be pairwise disjoint vertical columns of width $2^{-n^{\prime}}$. We define a sequence of measures $\left\{\tilde{\mu}_{k}\right\}_{k=1,2, \ldots}$ as follows: Recall that in the definition of $\mu_{k}, \mu_{k}$ is carried by $f^{k}(\operatorname{graph}(\alpha))$, where $f^{k}(\operatorname{graph}(\alpha))$ is a finite union of smooth curve segments. Let $\tilde{\mu}_{k}$ be $\mu_{k}$ annihilated on those parts of its support that only partially cross some $\tilde{U}_{i}$ (See Figure 3 .) $\tilde{\mu}_{k}(R)$ is probably $<1$.

We claim that given $\delta>0, \exists n^{\prime}$ such that for sufficiently large $k, \tilde{\mu}_{k}(R)>1-\delta$. Recall that $\exists M_{0}$ s.t. $\hat{g}_{k} \leq M_{0} \forall k$. If $x \in\left(\operatorname{supp} \mu_{k}-\operatorname{supp} \tilde{\mu}_{k}\right)$, then either $x$ lies in one of the two end pieces of $f^{k}(\operatorname{graph}(\alpha))$ that only partially crosses some $\tilde{U}_{i}$, or the horizontal distance between $x$ and a cusp in $f^{k}(\operatorname{graph}(\alpha))$ is $<2^{-n^{\prime}}$, which says that $d\left(f^{-i} x, S\right) \leq 2^{-n^{\prime}} u^{-i}$ for some $1 \leq i \leq k$. Thus

$$
\begin{aligned}
1-\tilde{\mu}_{k}(R) & =\mu_{k}\left(\operatorname{supp} \mu_{k}-\operatorname{supp} \tilde{\mu}_{k}\right) \\
& =\sum_{i=1}^{k} \mu_{k}\left\{x: d\left(f^{-i} x, S\right) \leq 2^{-n^{\prime}} u^{-i}\right\}+\mu_{k}\{2 \text { end pieces }\} \\
& =\sum_{i=1}^{k} \mu_{i}\left\{x: d(x, S) \leq 2^{-n^{\prime}} u^{-i}\right\}+\mu_{k}\{2 \text { end pieces }\} \\
& \leq 2 M_{0} q 2^{-n^{\prime}} \sum_{i=1}^{k} u^{-i}+\mu_{k}\{2 \text { end pieces }\}
\end{aligned}
$$

The second term $\rightarrow 0$ as $k \rightarrow \infty$. The first term becomes arbitrarily small as $n^{\prime} \uparrow \infty$. Recall also that $\left(n_{i}\right)^{-1} \sum_{k=1}^{n_{i}} \mu_{k} \rightarrow \mu$. Choose a subsequence $\left\{n_{i}^{\prime}\right\}$ of $\left\{n_{i}\right\}$ s.t. $\left(n_{i}^{\prime}\right)^{-1} \sum_{k=1}^{n_{i}^{\prime}} \tilde{\mu}_{k} \rightarrow \tilde{\mu}$ for some $\tilde{\mu}$. It is easy to verify that $\tilde{\mu} E \leq \mu E$ for every Borel set $E$ and hence we have $\tilde{\mu} \ll \mu$ with $0 \leq d \tilde{\mu} / d \mu \leq 1$. But since $\tilde{\mu}(R)$ can be made arbitrarily near 1 , we can choose $\tilde{\mu}$ s.t. $\tilde{\mu}$ is equivalent to $\mu$ except on a set of $\mu$-measure $<\varepsilon$.

It remains to show that if $\tilde{\mu}_{T}$ is the transverse measure on $P_{n^{\prime}}$ induced by $\tilde{\mu}$, then for $\tilde{\mu}_{T}$-a.e. $c \in P_{n^{\prime}} \mid V_{n^{\prime}}, \tilde{\mu}_{c} \ll m_{c}$. Recall that $g_{i_{1} \cdots i_{k}}=$ density of $p_{*}\left(\mu_{k} \mid L_{i_{1} \cdots i_{k}}\right)$. Let $\tilde{g}_{i_{1} \cdots i_{k}}=$ density of $p_{*}\left(\tilde{\mu}_{k} \mid L_{i_{1} \cdots i_{k}}\right)$. We claim that $\exists B>0$ s.t. for any $1 \leq i \leq 2^{n^{\prime}}$ 

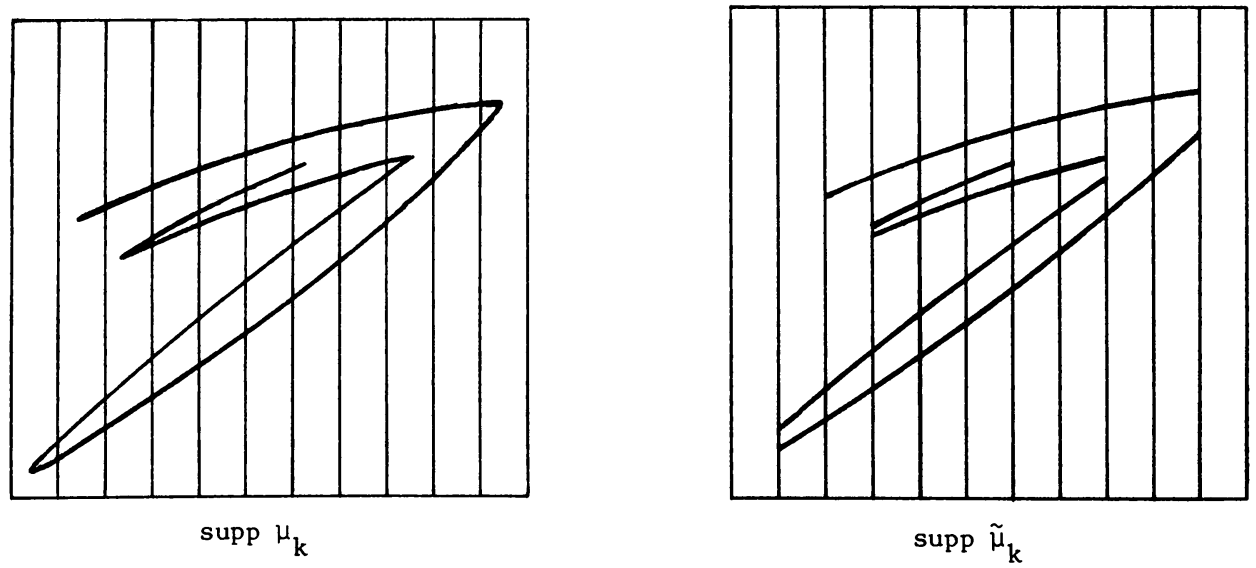

FIGURE 3

and any $i_{1} \cdots i_{k}$, we have either

$$
\tilde{g}_{i_{1} \cdots i_{k}} \equiv 0 \quad \text { on } p \tilde{U}_{i}
$$

or

$$
\frac{\tilde{g}_{i_{1} \cdots i_{k}}(x)}{\tilde{g}_{i_{1} \cdots i_{k}}(y)} \leq B \quad \forall x, y \in p \tilde{U}_{i} .
$$

This would prove that for almost every $c \in \mathcal{P}_{n^{\prime}} \mid V_{n^{\prime}}, d \tilde{\mu}_{c}=g_{c} d m_{c}$ where $\forall x, y \in c$, $g_{c}(x) / g_{c}(y) \leq B^{\prime}$ for some $B^{\prime}$. To prove the claim fix $1 \leq i \leq 2^{n^{\prime}}$ and $i_{1} \cdots i_{k}$. If $L_{i_{1} \cdots i_{k}}$ does not cross the full width of $\tilde{U}_{i}$, then $g_{i_{1} \cdots i_{k}} \mid p \tilde{U}_{i} \equiv 0$. Otherwise there are intervals $E_{0}, E_{1}, \ldots, E_{k} \subset[0,1], E_{k}=p \tilde{U}_{i}$, and $C^{2}$ diffeomorphisms $h_{j}: E_{j-1} \rightarrow E_{j}$ s.t. $\tilde{g}_{i_{1} \cdots i_{k}}\left|p \tilde{U}_{i}=\right|\left(\left(h_{k} \circ \cdots \circ h_{1}\right)^{-1}\right)^{\prime} \mid$ (see lemma). While these $h_{j}$ 's depend strictly on $i$ and $i_{1} \cdots i_{k}$, they satisfy $\left|h_{j}^{\prime}\right| \geq u>1$ and $\left|h_{j}^{\prime \prime}\right| \leq Q$, where $u$ and $Q$ are universal constants.

The reader can easily verify that

$$
\begin{aligned}
& |\log |\left(\left(h_{k} \circ \cdots \circ h_{1}\right)^{-1}\right)^{\prime}(x)|-\log |\left(\left(h_{k} \cdots h_{1}\right)^{-1}\right)^{\prime}(y)|| \\
& \quad \leq \text { some constant depending only on } u, Q \text { and } n^{\prime} .
\end{aligned}
$$

This completes the proof.

We have proved our result for a specific class of maps of the square into itself. It is obvious that our hypotheses are more stringent than necessary and that it is easy to make slight generalizations. We do not attempt to do that here, because we do not know what a natural general statement ought to be.

We mention a few corollaries. Since the proofs are standard, we will provide only references. The standing hypothesis for the rest of this article is that $f$ is a generalized Lozi map.

COROLlaRY 1. Let $\mu$ be constructed as in our lemma. Then there are measurable sets $E_{1}, E_{2}, \cdots \subset R$ s.t. $f^{-1} E_{i}=E_{i}, \mu E_{i}>0 \forall i, \mu\left(\bigcup E_{i}\right)=1$ and for each $i, f \mid E_{i}:\left(E_{i}, \mu \mid E_{i}\right) \rightarrow\left(E_{i}, \mu \mid E_{i}\right)$ is ergodic.

Proof. The proof follows that in $[\mathbf{P}]$. It uses the absolute continuity of stable manifolds $[\mathbf{K S}]$ and the fact that on most $c \in \mathcal{P}_{n} \mid V_{n}, \mu_{c}$ is equivalent to $m_{c}$. 
Definition 2. A Borel probability measure $\mu$ on $R$ is called a Bowen-Ruelle measure $[\mathbf{B}]$ if there is a set $U \subset R$ of positive Lebesgue measure s.t. for every continuous function $\varphi: \mathbf{R} \rightarrow \mathbf{R}$,

$$
\frac{1}{n} \sum_{i=0}^{n-1} \varphi f^{i} x \rightarrow \int \varphi d \mu
$$

for Lebesgue-a.e. $x \in U$.

COROLlaRY 2. Let $\mu$ be constructed as in our lemma. Then corresponding to each $E_{i}$ in Corollary 1, $\mu \mid E_{i}$ normalized is a Bowen-Ruelle measure.

PROOF. This follows from $[\mathbf{K S}]$.

Unlike the case of Axiom A attractors, assuming only (H1)-(H4) there can easily be more than one Bowen-Ruelle measure.

COROLlary 3. Let $f: R \rightarrow R$ be a generalized Lozi map. Then $f$ has an invariant Borel probability measure $\mu$ s.t.

$$
h_{\mu}(f)=\int \lambda_{1}(x) d \mu(x),
$$

where $\lambda_{1}(x)$ is the positive $\mu$-exponent of $f$ at $x$.

PROOF. See $[\mathbf{L S}]$.

\section{REFERENCES}

[B] R. Bowen, Equilibrium states and the ergodic theory of Anosov diffeomorphisms, Lecture Notes in Math., vol. 47, Springer, Berlin and New York, 1975.

[CL] P. Collet and Y. Levy, Ergodic properties of the Lozi mappings, Comm. Math. Phys. 93 (1984), 461-482.

[KS] A. Katok and J. M. Strelcyn, Invariant manifolds for smooth maps with singularities, Part I: Existence (preprint).

[LS] F. Ledrappier and J. M. Strelcyn, A proof of the estimation from below in Pesin entropy formula, J. Ergodic Theory and Dynam. Syst. (to appear).

[LY] A. Lasota and J. Yorke, On the existence of invariant measures for piecewise monotonic transformations, Trans. Amer. Math. Soc. 186 (1973), 481-488.

[M] M. Misiurewicz, Strange attractors for the Lozi mappings, Proc. N.Y. Acad. Sci. (1980), 348-358.

[P] Ja. Pesin, Characteristic Lyapunov exponents and smooth ergodic theory, Russian Math. Surveys 32 (1977), 55-114.

[S] Ya. G. Sinai, Markov partitions and C-diffeomorphisms, Functional Anal. Appl. 2 (1968), 61-82.

Department of Mathematics, Michigan State University, East lansing, MICHIGAN 48824 (Current address)

Department of Mathematics, University of North Carolina, Chapel Hill, NORTH CAROLINA 27514 